\title{
Derivation of the semicircle law from the law of corresponding states
}

\author{
C. P. Burgess, ${ }^{1, *}$ Rim Dib,$^{1, \dagger}$ and Brian P. Dolan ${ }^{2, \ddagger}$ \\ ${ }^{1}$ Physics Department, McGill University, 3600 University Street, Montréal, Québec, Canada H3A $2 T 8$ \\ ${ }^{2}$ Groupe de Physique Théorique, Institut de Physique Nucléaire, F-91406 ORSAY Cedex, France
}

(Received 7 August 2000)

\begin{abstract}
We show that, for the transition between any two quantum Hall states, the semicircle law and the existence of a duality symmetry follow solely from the consistency of the law of corresponding states with the twodimensional scaling flow. This puts these two effects on a sound theoretical footing, implying that both should hold exactly at zero temperature, independently of the details of the microscopic electron dynamics. This derivation also shows how the experimental evidence favors taking the two-dimensional flow seriously for the whole transition, and not just near the critical points.
\end{abstract}

Quantum Hall systems are remarkable for the high accuracy with which many of their properties are known. Although an explanation based on general principles (gauge invariance) has been given by Laughlin ${ }^{1}$ for the precise quantization of the Hall conductivity on the integer Hall plateaus, to our knowledge, a similar understanding of the robustness of the other Hall features does not yet exist. Our goal in this paper is to derive some of these as general consequences of the symmetries of the low-energy limit of these systems, independently of the microscopic details. In particular, we show how to do this for two remarkable experimentally observed effects: the semicircle law and the duality $\rho_{x x} \rightarrow 1 / \rho_{x x}$ in the plateau/insulator transition, which we here argue follow purely as consequences of a symmetry that has been proposed to hold for quantum Hall systems. The utility of relating these effects to a symmetry is that it makes their theoretical interpretation much cleaner. These effects should be expected to occur for any systems that fall into the domain of validity of the symmetry in question.

The current understanding of the transport properties of quantum Hall systems is based on a very successful effective field theory consisting of composite fermions. ${ }^{2}$ The symmetry that we shall use in what follows was argued to be a property of the effective theory, under certain circumstances, in a seminal paper by Kivelson, Lee, and Zhang. ${ }^{3}$ These authors argue that the effective theory satisfies a law of corresponding states, which consist of the following correspondences between conductivities, in the long-wavelength limit: Landau-level addition transformation $(\mathbf{L})$,

$$
\sigma_{x y}(\nu+1) \leftrightarrow \sigma_{x y}(\nu)+1 \quad \sigma_{x x}(\nu+1) \leftrightarrow \sigma_{x x}(\nu) ;
$$

flux attachment transformation $(\mathbf{F})$,

$$
\rho_{x y}\left(\frac{\nu}{2 \nu+1}\right) \leftrightarrow \rho_{x y}(\nu)+2 \quad \rho_{x x}\left(\frac{\nu}{2 \nu+1}\right) \leftrightarrow \rho_{x x}(\nu) ;
$$

particle-hole transformation $(\mathbf{P})$

$$
\sigma_{x y}(1-\nu) \leftrightarrow 1-\sigma_{x y}(\nu) \quad \sigma_{x x}(1-\nu) \leftrightarrow \sigma_{x x}(\nu) ;
$$

where $\nu$ denotes the filling factor and we use units in which $e^{2} / h=1$.

The arrows become equalities when the correspondence becomes a symmetry, and the conditions for this to be the case are discussed in Ref. 3-in particular this is expected to hold at zero temperature. Taking repeated powers of $\mathbf{L}, \mathbf{F}$, and their inverses generates an infinite order discrete group, which we shall denote by $\mathcal{K}$, which is well known to mathematicians [see, e.g., Ref. 4 where it is denoted by $\left.\Gamma_{U}(2)\right]$. The complete group, including also the transformation $\mathbf{P}$, which is an outer automorphism of $\mathcal{K}$, was first proposed as relevant to the quantum Hall effect by Lütken and Ross. ${ }^{5}$

The symmetry $\mathcal{K}$ is most succinctly expressed by writing the conductivity tensor as a single complex variable, $\sigma$ $=\sigma_{x y}+i \sigma_{x x}$ (with the resistivities therefore given by $\rho=$ $\left.-1 / \sigma=-\rho_{x y}+i \rho_{x x}\right)$. Since the Ohmic resistance $\sigma_{x x}$ must be positive, the physical region consists only of the upperhalf $\sigma$ plane. A general element of $\mathcal{K}$ can then be represented as $\gamma(\sigma)=(a \sigma+b) /(c \sigma+d)$ with integer coefficients, such that $c$ is even and $a d-b c=1$. Note that $\mathcal{K}$ maps the upper half-complex conductivity plane into itself.

The whole upper-half conductivity plane can be obtained from the vertical strip above the semicircle of unit diameter spanning 0 and 1 by repeated action of $\mathcal{K}$. This strip is termed the fundamental domain in the mathematical literature.

The law of corresponding states can now be seen to imply that any quantum Hall state can be obtained from any other state by the action of some element of $\mathcal{K}$. Thus, for example, starting from $\sigma=1$ we obtain the integer series $\sigma=n$ from $\mathbf{L}^{n-1}$, the Laughlin series $\sigma=1 /(2 m+1)$ from $\mathbf{F}^{m}$, and the Jain series $\sigma=p /(2 p m+1)$ from $\mathbf{F}^{m} \mathbf{L}^{p-1}$. It has already been pointed out ${ }^{12}$ that $\mathcal{K}$ gives a selection rule for quantum Hall transitions - a transition between two Hall plateaus with $\sigma_{x y}=p_{1} / q_{1}$ and $\sigma_{x y}=p_{2} / q_{2}$ is allowed only if $\mid p_{1} q_{2}$ $-p_{2} q_{1} \mid=1$. However, it implies much more if we examine the consequences for the $\beta$ functions of the theory.

Strong predictions can be made when the symmetry $\mathcal{K}$ is combined with the scaling theory of disorder, ${ }^{6}$ as applied to quantum Hall systems. $^{7-9}$ According to the scaling theory, conductances (so, in two dimensions, also the conductivities) are the macroscopic measures of microscopic disorder, and so are the important variables whose RG flow describes the system's long-wavelength evolution. For quantum Hall systems this implies a two-dimensional description of the flow, since both $\sigma_{x x}$ and $\sigma_{x y}$ can play a role. The flow in this 
two-dimensional plane has various fixed points, and in general which combination of $\sigma_{x x}$ or $\sigma_{x y}$ is RG relevant will depend on the fixed point that is under consideration. [For instance, although both $\sigma_{x x}$ and $\sigma_{x y}$ are irrelevant (in the infrared) at the fixed points corresponding to the Hall plateaus, both are relevant at the repulsive fixed points, such as the one at $\left(\sigma_{x x}, \sigma_{x y}\right)=\left(0, \frac{1}{2}\right)$ in the flow diagram in Ref. 7. Both are clearly important when discussing the crossover between different fixed points.] The basic assumption made by such a scaling theory, and which we also make, is that the long-wavelength RG flow of the system flows much more slowly along this two-dimensional surface, than is the flow to this surface through the many-dimensional space parametrized by the many irrelevant microscopic degrees of freedom. The scaling theory literature has proven the utility of this hypothesis.

In this language the law of corresponding states becomes the requirement that $\mathcal{K}$ (and $\mathbf{P}$ ) should commute with the $\mathrm{RG}$ flow. ${ }^{10-12}$ The flow is described by a single complex $\beta$ function:

$$
\beta(\sigma, \bar{\sigma})=\frac{d \sigma}{d t}=\beta_{x y}\left(\sigma_{x x}, \sigma_{x y}\right)+i \beta_{x x}\left(\sigma_{x x}, \sigma_{x y}\right),
$$

and a simple calculation reveals that the flow commutes with the symmetry if ${ }^{11}$

$$
\beta(\gamma(\sigma), \gamma(\bar{\sigma}))=\frac{d \gamma(\sigma)}{d t}=\frac{\beta(\sigma, \bar{\sigma})}{(c \sigma+d)^{2}},
$$

where the property $a d-b c=1$ has been used.

We now describe some consequences that follow for the flow of any $\beta$ function that satisfies Eq. (2) (and subject to a global requirement concerning flow topology, as explained below) regardless of its detailed form. Previous analyses have made further assumptions about the functional form of $\beta,{ }^{11,13-16}$ but we shall avoid any such assumptions here and simply follow the implications of particle-hole symmetry.

It is an immediate consequence of Eq. (2) that the $\beta$ function must vanish at any point $\sigma_{*}$ (called a fixed point) that is taken to itself-i.e., $\gamma\left(\sigma_{*}\right)=\sigma_{*}$-by the action of a group element for which $c \sigma_{*}+d$ is neither zero nor infinite. ${ }^{5,11}$ The only such fixed points within the fundamental domain are the one at $\sigma_{*}=\frac{1}{2}(1+i)$-which is taken to itself by $\gamma(\sigma)=(\sigma-1) /(2 \sigma-1)$-as well as $\sigma=0$-with $\gamma(\sigma)=$ $-\sigma /(2 \sigma-1)$-and $\quad \sigma=1$-with $\quad \gamma(\sigma)=(3 \sigma-2) /(2 \sigma$ $-1)$. $\beta$ must therefore vanish at these three points (assuming it is finite). The consistency of $\mathcal{K}$ with the flow thereby predicts universal values for the conductivity at the critical points, a possibility that was argued within a more general context in Ref. 17.

The symmetry $\mathcal{K}$ also requires the $\beta$ function to vanishand to have precisely the same critical exponents - at all of the images of the basic fixed points under the action of $\mathcal{K}$. There is indeed experimental evidence for this equivalence of the critical exponents at different quantum Hall transitions (known as superuniversality), ${ }^{18,19}$ a result that had been also argued microscopically (neglecting electron self-interactions). ${ }^{20}$

It is not an inescapable consequence of $\mathcal{K}$ that there be no critical points other than those that are fixed points of $\mathcal{K}$.
However, there is no experimental evidence for any other critical points in the quantum Hall effect, and so it would not seem unreasonable to assume there is none. None of the following conclusions requires this assumption unless explicitly stated.

Most of the above observations have already appeared in the literature but we now go on to describe the two main results of this paper, which have not been derived from general principles before.

\section{The semicircle law}

We now show that particle-hole symmetry, together with $\mathcal{K}$ invariance, implies the semicircle law. The proof of this argument hinges on the existence of a unique and wellknown function, $f(\sigma)$, which has the following two crucial properties: ${ }^{4}$ (1) It provides a one-to-one map from the fundamental domain to the complex plane (including the point at infinity); (2) it is invariant under the action of the symmetry group $\mathcal{K}, f(\gamma(\sigma))=f(\sigma)$. Define a $\beta$ function for $f$,

$$
B(f, \bar{f}):=\frac{d f}{d t}=\frac{d f}{d \sigma} \frac{d \sigma}{d t}=\frac{d f}{d \sigma} \beta(\sigma, \bar{\sigma}) .
$$

We can conclude that $B(f, \bar{f})$ is invariant with respect to $\mathcal{K}$-since $f$ is already invariant, $\mathcal{K}$ invariance imposes no further restrictions on the function $B(f, \bar{f})$.

Now impose particle-hole symmetry $\mathbf{P}$. To determine the consequences for $B(f, \bar{f})$ due to $\mathbf{P}$ we use the following explicit expression $^{4}$ for $f$ in terms of Jacobi $\vartheta$ functions (a very clear description of these classical functions is given in Ref. 21):

$$
f(\sigma)=-\frac{\vartheta_{3}^{4} \vartheta_{4}^{4}}{\vartheta_{2}^{8}}=-\frac{1}{256 q^{2}} \prod_{n=1}^{\infty} \frac{\left(1-q^{4 n-2}\right)^{8}}{\left(1+q^{2 n}\right)^{16}},
$$

where $q=e^{i \pi \sigma}$. Since the action of particle-hole symmetry on $q$ is $\mathbf{P}: q \rightarrow-\bar{q}$, it is clear from the definition of $f$ that $\mathbf{P}: f=f(-\bar{q})=\overline{f(q)}$. Thus particle-hole symmetry implies that $B(f, \bar{f})$ must be invariant under the interchange of $f$ and $\bar{f}$. So this implies that

$$
\frac{d f}{d t}=B(f, \bar{f}), \quad \frac{d \bar{f}}{d t}=\overline{B(f, \bar{f})}=B(\bar{f}, f) .
$$

Now suppose we start our RG flow from a value of $\sigma$ for which $f$ is real. Equation (5) states that $B$ is real when evaluated at this point, and hence $d f / d t$ must be real. Repeating this argument point by point along the flow line we see that particle-hole symmetry implies $f$ cannot develop an imaginary part if it does not start with one. We conclude that curves on which $f$ is real are integral curves of any renormalization-group flow that commutes with both $\mathcal{K}$ and P.

The curves along which $f$ is real are easily found, and for the fundamental domain consist of the curves defining the boundaries, plus the vertical line $\sigma=\frac{1}{2}+i w, w \geqslant \frac{1}{2} . f$ is real along the vertical lines $\sigma=n / 2+i w$ (with $n$ integral) because it is an even function of $q$, and $q$ is real or pure imaginary when evaluated along these vertical lines. To see that $f$ is 


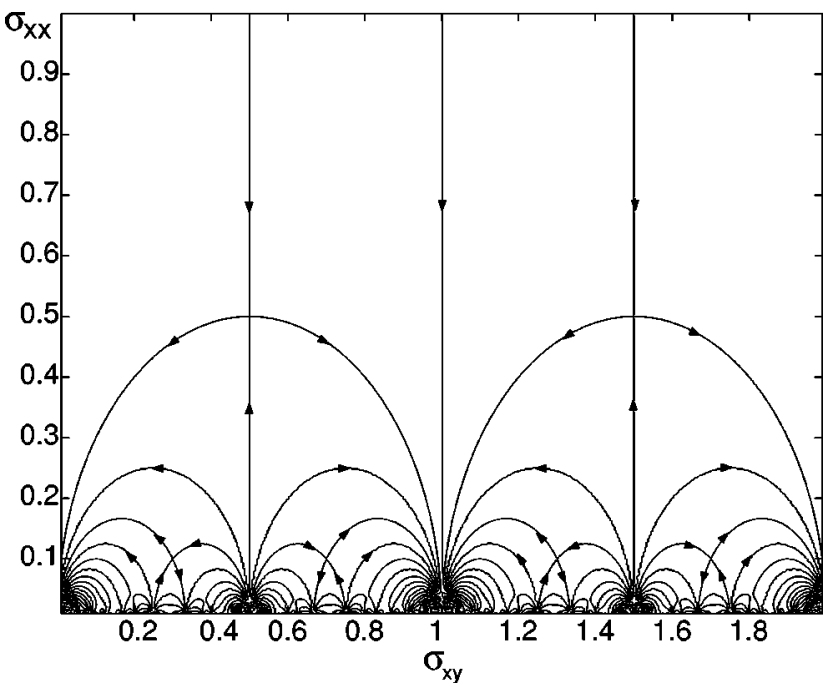

FIG. 1. Crossover flow of the conductivities as predicted from the law of corresponding states.

also real on the semicircular arch spanning 0 and 1 requires the following classical facts about the $\vartheta$ functions (see, e.g., Ref. 21, p. 475):

$$
\vartheta_{2}(-1 / \sigma)=\sqrt{-i \sigma} \vartheta_{4}(\sigma), \quad \vartheta_{3}(-1 / \sigma)=\sqrt{-i \sigma} \vartheta_{3}(\sigma),
$$

$$
\vartheta_{4}(-1 / \sigma)=\sqrt{-i \sigma} \vartheta_{2}(\sigma)
$$

The factors of $\sqrt{-i \sigma}$ here cancel when we take ratios to form $f$ and so $f(-1 / \sigma)=-\vartheta_{3}^{4}(\sigma) \vartheta_{2}^{4}(\sigma) / \vartheta_{4}^{8}(\sigma)$. Now $\sigma \rightarrow$ $-1 / \sigma$ sends the vertical line $\sigma=1+i w, 0<w<\infty$, onto the semicircle spanning 1 and 0 . Since $\vartheta_{a}^{4}(a=2,3,4)$ are all real on said vertical line (see, e.g., Ref. 21), $f$ must be real on said semicircle, the latter is then perforce an integral curve of the renormalization-group flow.

The complete set of integral curves is then obtained by mapping the above curves around the complex plane using $\mathcal{K}$, and this is how Fig. 1 is generated. Points where trajectories cross are fixed points of the renormalization-group flow, and the fixed point at $\sigma_{*}=(1+i) / 2$ is evident. The direction of the flow lines is uniquely determined if we assume that there are no other fixed points, the Hall plateaus are attractive fixed points of the flow, and that the flow comes downwards vertically from $\sigma=i \infty$. The line segment $\sigma=-\frac{1}{2}+i w, \quad \frac{1}{2}<w<\infty$ is mapped onto $\sigma=\frac{1}{2}+i w, 0<w$ $<\frac{1}{2}$ by $\mathbf{F}$ - the latter line must therefore flow upwards towards $(1+i) / 2$ if the former flows downwards towards $(-1+i) / 2$. Assuming that 0 and 1 are attractive fixed points then determines the flow direction as indicated by the arrows in Fig. 1. Notice we are inevitably led to the existence of the semicircles linking 0 to $1 / 2$ in Fig. 1 .

It is a general property that $\mathcal{K}$ takes semicircles centered on the real axis to other semicircles also centered on the real axis (including the degenerate case of infinitely large semicircles, which are vertical lines parallel to the imaginary axis). It follows that the flow between any two Hall plateaus must be along a semicircle, centered on the real axis, which is the image of the basic semicircle connecting 0 and 1 .
In this way we obtain a robust derivation of the semicircle law, which states that the conductivities move along such semicircles in the conductivity plane during transitions between Hall plateaus. (Since the relation $\rho=-1 / \sigma$ maps, for example, semicircles having one end at $\sigma=0$ into vertical lines in the $\rho$ plane, the corresponding statement in the resistivity plane is that for transitions from Hall fluids to the Hall insulator, the flow is along lines of constant $\rho_{x y}$.) Although the semicircle law was proposed in Ref. 22 on the basis of a particular microscopic model, we see here that it holds more generally than does its original derivation. Any model compatible with the symmetry of the law of corresponding states must reproduce it. Experimentally, the law is also well supported. ${ }^{23}$

\section{Duality}

A second experimentally striking result that follows quite generally from the symmetry version of the law of corresponding states is the existence of a duality symmetry relating the conductivities of the flow on either side of the critical point as one flows between any two allowed Hall plateaus, or between Hall plateaus and the Hall insulator.

Since all flows are related by a symmetry to the basic semicircle running between 0 and 1, we derive the duality symmetry for this semicircle in detail. A convenient parametrization of the semicircle from 0 to 1 is

$$
\sigma=\frac{1}{2}+\frac{1}{2}\left(\frac{1-w^{2}+2 i w}{1+w^{2}}\right),
$$

with $0<w<\infty$. The key observation is that this curve is reflected into itself about the vertical line $\Re \sigma=\frac{1}{2}$ by $\mathbf{P}$-as well as by $\gamma=(\sigma-1) /(2 \sigma-1) \in \mathcal{K}$. In terms of the parameter $w$ this becomes $w \rightarrow 1 / w$, and since the semicircle transformed to the $\rho$ plane is $\rho=-1+i w$, this is recognizable as the $\rho_{x x} \rightarrow 1 / \rho_{x x}$ duality that has been observed ${ }^{24}$ in the transition to the Hall insulator from the $\nu=1$ integer Hall state.

The extension to other transitions follows from the action of $\mathcal{K}$. For $\nu: p_{1} / q_{1} \rightarrow p_{2} / q_{2}$ with $p_{2} q_{1}-p_{1} q_{2}=1$, where the transition is along the curve $\rho=\left[-\left(q_{2} p_{2}+w^{2} q_{1} p_{1}\right)\right.$ $+i w] /\left(p_{2}^{2}+w^{2} p_{1}^{2}\right)$, the duality is again given by $w \rightarrow 1 / w$. As specialized to transitions to the Hall insulator from the Laughlin sequence, $\nu: 1 /(2 n+1) \rightarrow 0$, the flow is the vertical line $\rho=-(2 n+1)+i w$ in the resistivity plane and so the duality $w \rightarrow 1 / w$ again implies the inversion $\rho_{x x} \rightarrow 1 / \rho_{x x}$ about the critical point.

In conclusion, we wish to emphasize two points. First, the assumption that the law of corresponding states holds as a symmetry at low temperatures leads to an infinite order discrete symmetry group for the quantum Hall effect-called $\mathcal{K}$ here. This group acts on the upper-half complex conductivity plane. If this is to be a symmetry its action must commute with the renormalization-group flow of the system and fixed points of the group action must be fixed points of the flow. Three kinds of fixed points are predicted in this way: attractive fixed points with $\sigma_{x x}=0$ (which are images under the group of the basic ones at $\sigma=0$ or 1 and all have odd denominator) describing the quantum Hall fluids and the Hall insulator; repulsive fixed points with $\sigma_{x x}=0$ (which are images of $\sigma=1 / 2$ and all have even denominator); and saddle points [which are images under the group of the basic one at 
$\left.\sigma=\frac{1}{2}(1+i)\right]$ describing the critical points in the transitions between the various quantum Hall states. By organizing the critical points of the system via a (infinite order) discrete symmetry, the group $\mathcal{K}$ furnishes a fascinating generalization of the Kramers-Wannier $\mathbf{Z}_{2}$ duality of the Ising model. The symmetry also inescapably predicts the general existence of a duality symmetry for all Hall transitions, which reduces to the observed $\rho_{x x} \rightarrow 1 / \rho_{x x}$ duality for Laughlin-sequence/Hallinsulator transitions.

Particle-hole symmetry places further restrictions on the $\beta$ function and dictates that the form of the RG flow between Hall plateaus, and between the plateaus and the Hall insulator, be described by semicircle law.

A point that must be addressed here is that the experimental data do not always reproduce a fixed point exactly at $\sigma_{x x}=1 / 2$ for integer transitions. For example, in Ref. 23 the critical point in the $1 \rightarrow 0$ transition is definitely not identified with $(1+i) / 2$. This is therefore incompatible with the law of corresponding states. However, it is notoriously difficult to extract the longitudinal resistivity (and hence conductivity) from the longitudinal resistance (this issue is discussed, for example, on p. 52 in Cage's paper in Ref. 25) - the relationship between these two quantities is plagued with geometrical ambiguities, unlike the transverse resistivity. Since a Hall-insulator transition $1 /(2 q+1) \rightarrow 0$ in the $\sigma$ plane corresponds to a vertical line $\rho=-(2 q+1)+i w, w>0$, in the resistivity plane, rescaling the imaginary part of $\rho$ does not affect the semicircle law in the $\sigma$ plane for these transitions. However it does move the critical point. One interpretation of the experiment ${ }^{23}$ is that the experimentally determined longitudinal resistivity is not the same as the true microscopic longitudinal resistivity, but a constant multiple of it (a factor of 1.7 in this particular experiment).

We thank C. A. Lütken for helpful discussions. C.P.B. is grateful to the University of Barcelona, and B.P.D. to the IPN, Orsay and CNRS, for their generous support and kind hospitality while part of this work was carried out. Research funds for this work have been provided by grants from NSERC (Canada), FCAR (Québec), CNRS (France), and by Enterprise Ireland (Basic Research Grant No. SC/1998/739).
*Email address: cliff@physics.mcgill.ca

†Email address: rimdib@physics.mcgill.ca

†n leave from Dept. of Mathematical Physics, National University of Ireland, Maynooth, Republic of Ireland. Email address: bdolan@thphys.may.ie

${ }^{1}$ R. Laughlin, Phys. Rev. B 23, 5632 (1981).

${ }^{2}$ J. K. Jain, Phys. Rev. Lett. 63, 199 (1989); Adv. Phys. 41, 105 (1992).

${ }^{3}$ S. Kivelson, D-H. Lee, and S.-C. Zhang, Phys. Rev. B 46, 2223 (1992).

${ }^{4}$ R. A. Rankin, Modular Forms and Functions (Cambridge University Press, Cambridge, 1977).

${ }^{5}$ C. A. Lütken and G. G. Ross, Phys. Rev. B 45, 11837 (1992); 48, 2500 (1993).

${ }^{6}$ E. Abrahams, P. W. Anderson, D. C. Licciardello, and T. V. Ramakrishnan, Phys. Rev. Lett. 42, 673 (1979).

${ }^{7}$ D. E. Khmel'nitskii, Pis'ma Zh. Éksp. Teor. Fiz. 38, 454 (1983) [JETP Lett. 38, 552 (1983)].

${ }^{8}$ A. M. M. Pruisken, Phys. Rev. Lett. 61, 1297 (1988).

${ }^{9}$ B. Huckelstein, Rev. Mod. Phys. 67, 357 (1995); S. L. Sondhi, S. M. Girvin, J. P. Carini, and D. Shahar, ibid. 69, 315 (1997).

${ }^{10}$ C. A. Lütken, Nucl. Phys. B 396, 670 (1993).

${ }^{11}$ C. P. Burgess and C. A. Lütken, Nucl. Phys. B 500, 367 (1997).

${ }^{12}$ B. P. Dolan, J. Phys. A 32, L243 (1999).
${ }^{13}$ B. P. Dolan, Nucl. Phys. B: Field Theory Stat. Syst. 460, [FS] 297 (1999).

${ }^{14}$ N. Taniguchi, cond-mat/9810334 (unpublished).

${ }^{15}$ C. P. Burgess and C. A. Lütken, Phys. Lett. B 451, 365 (1999).

${ }^{16}$ Y. Georgelin, T. Masson, and J-C. Wallet, J. Phys. A 33, 39 (2000).

${ }^{17}$ M. P. A. Fisher, G. Grinstein, and S. M. Girvin, Phys. Rev. Lett. 64, 587 (1990); M.-C. Cha, M. P. A. Fisher, S. M. Girvin, M. Wallin, and A. P. Young, Phys. Rev. B 44, 6883 (1991).

${ }^{18}$ H. P. Wei, D. C. Tsui, M. A. Paalanen, and A. M. M. Pruisken, Phys. Rev. Lett. 61, 1294 (1988).

${ }^{19}$ L. Engel et al., Surf. Sci. 229, 13 (1990).

${ }^{20}$ D. E. Khmel'nitskii, in Ref. 7; A. M. M. Pruisken, in Refs. 8 and 25; R. B. Laughlin, Phys. Rev. Lett. 52, 2304 (1984).

${ }^{21}$ E. T. Whittaker and G. N. Watson, A Course of Modern Analysis (Cambridge University Press, Cambridge, 1940).

${ }^{22}$ A. M. Dykhne and I. M. Ruzin, Phys. Rev. B 50, 2369 (1994); I. Ruzin and S. Feng, Phys. Rev. Lett. 74, 154 (1995).

${ }^{23}$ M. Hilke et al., Europhys. Lett. 46, 775 (1999).

${ }^{24}$ D. Shahar, D. C. Tsui, M. Shayegan, J. E. Cunningham, E. Shimshoni, and S. L. Sondhi, Solid State Commun. 102, 817 (1997); D. Shahar, D. C. Tsui, M. Shayegan, E. Shimshoni, and S. L. Sondhi, Science 274, 589 (1996).

${ }^{25}$ The Quantum Hall Effect, edited by R. E. Prange and S. M. Girvin (Springer, Berlin, 1987). 